\title{
NUMERICAL INVESTIGATION OF ACOUSTIC CHARACTERISTICS OF 3D HUMAN VOCAL TRACT MODEL WITH NASAL CAVITIES
}

\author{
T. Vampola*, J. Štorkán*, J. Horáček**, V. Radolf **
}

\begin{abstract}
Acoustic resonance characteristics of 3D human vocal tract model without and with nasal and paranasal cavities were computed. Nasal cavities (NC) form the side branches of the human vocal tract and exhibit antiresonance and resonance properties which influence the produced voice quality. Developed FE models of acoustic spaces of nasal and vocal tract for vowel /a:/ are used to study the influence of (NC) on phonation. Acoustics frequency-modal characteristics are studied by modal analysis and numerical simulation of acoustic signals in time domain is performed by transient analysis of the FE models.
\end{abstract}

Keywords: formant frequencies, modelling of phonation, biomechanics of voice

\section{Introduction}

Human voice is based on self-oscillations of the vocal folds excited by air flowing from the lungs. The vibration of the vocal folds modulates the stream of air producing the primary sound of voice in a narrow oscillating constriction between the two vocal folds called glottis. This primary sound signal propagates through the supralaryngeal cavities between the vocal folds, lips and the nostrils (i.e., the vocal tract) which modify its quality through acoustic resonances. The acoustic resonances of the vocal tract create the so-called formants, which occur as peaks in the envelope of the voice spectrum. The formants are determined by the size and shape of the vocal tract cavities and define vowels and the voice timbre. The final sound quality of human voice is thus given both by the characteristics of the vocal fold vibration and by the vocal tract properties (Sundberg 1987).

While the influence of the geometric configuration of the main channel of the vocal tract on the vocal output has been studied rather extensively, the influence of side cavities of human vocal tract has received less attention. As such, their role for the resulting vocal intensity may be considered undesirable, since it contradicts the general goal of enhancing vocal output with the smallest vocal effort. A more detailed analysis shows that besides the antiresonances there are also new resonances which occur due to these side cavities. The effects of nasal cavities are studied in the present paper, where the nasality or so-called velopharyngeal insufficiency is modeled by interconnecting the acoustic cavities of the nasal tract with the vocal tract at the velum (soft palate). If the velum is raised properly the nasal tract is closed and the interconnection is shut. If the velum muscles are flabbier the interconnecting space between the vocal tract and nasal tract is getting bigger. Especially, this effect is studied here in relation to the changes of the lowest formant frequencies. Similar study was performed by the authors in the previous paper (Vampola et al. 2008), where the velopharyngeal insufficiency and clefting of soft or hard palate were studied in relation to the voice quality for the vowels [/a:/,ii:/,u:/]. The influence of clefting on phonation for vowel /a:/ was found smaller than the influence of the clefts on the pronunciation and perception of vowels /i:/ and /u:/. However, the models of the human vocal and nasal tracts used in the previous study were very much simplified, and for a proper prediction of the voice quality it is necessary to use more

\footnotetext{
* prof. Dr. Ing. Tomáš Vampola, Ing. Jaroslav Štorkán: Faculty of Mechanical Engineering, Czech Technical University in Prague; Technická 4; 166 07, Prague; CZ, Email: tomas.vampola@fs.cvut.cz

** Ing. Jaromír Horáček, DrSc., Ing. Vojtěch Radolf, PhD.: Institute of Thermomechanics, The Czech Academy of Sciences, Dolejškova 1402/5; 182 00, Prague; CZ, Email: jaromirh@it.cas.cz, radolf@it.cas.cz
} 
correct models, particularly of the nasal cavities. Here we are concentrated on modelling the phonation for vowel /a:/ with and without nasality.

\section{Methods}

Three-dimensional (3D) model of the vocal tract (VT) for the vowel /a:/ was created from the Computer Tomography (CT) measurement of a female subject during phonation, see (Vampola et al., 2015). The complex 3D volume model of acoustic nasal cavities was developed from a detailed CT investigation of the head of another subject of the same gender, similar age and size. After segmentation of the CT images we obtained the volume model of the nasal tract interconnected with the volume model of the vocal tract for the vowel /a:/, see Fig.1.
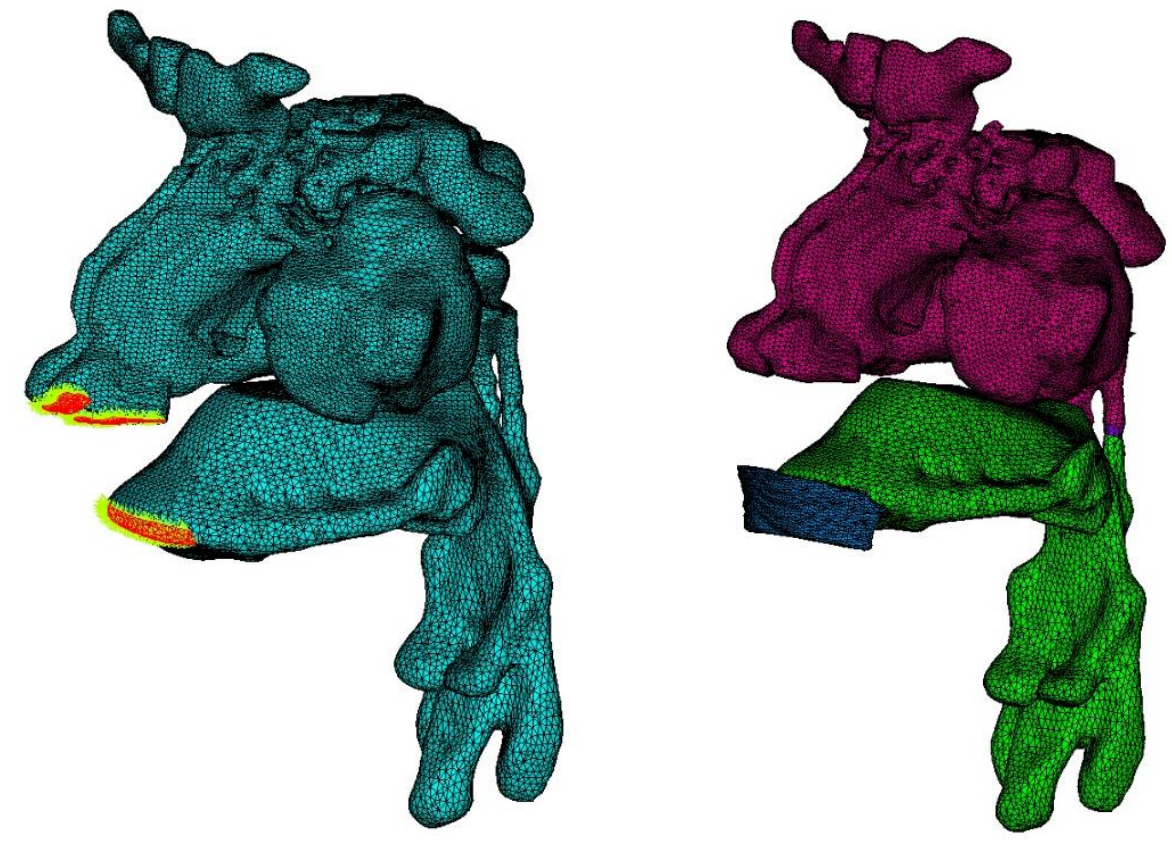

Fig. 1 Computational model of the human vocal tract for vowel /a:/ with nasal cavities without considering the lips (left) and with the lips (right).

Acoustic energy losses in the vocal tract, modelled by the sound radiation from the mouth and nose to open atmosphere, belong to main acoustic energy dissipation losses in the vocal tract. The radiation losses were modelled by a circular plate vibrating like a piston in an infinite wall, for which the following frequency dependent acoustic impedance can be derived (Vampola et al. 2008)

$$
Z_{a}=\frac{c_{0} \rho_{0}}{S}(A+i B), \quad A=1-\frac{J_{1}(2 k R)}{k R}, \quad B=\frac{H_{1}(2 k R)}{k R},
$$

where $c_{0}$ is sound velocity $(346 \mathrm{~m} / \mathrm{s}), \rho_{0}(1.2 \mathrm{~kg} / \mathrm{m} 3)$ is air density, $\mathrm{R}$ is equivalent radius of a vibrating plate calculated from the cross-section of the vocal tract model at the lips level and at the nostrils, $\mathrm{k}=$ $\omega / c_{0}$ is wave number, $\omega$ is angular frequency and $\mathrm{i}$ is imaginary unit. The Bessel $J_{l}$ and Struve $H_{l}$ functions can be calculated using the infinitive series. The acoustic energy losses inside the vocal tract due to, e.g., air viscosity and flexibility of the soft tissues on the boundaries of the acoustic spaces, were incorporated in the model via the boundary admittance coefficient $\mu=r / \rho_{0} c_{0}$, where $r$ is the real component of the specific acoustic impedance (resistance term). Boundary admittance coefficient $\mu \in(0$; 1 ) is related to the dimensionless absorption coefficient $\alpha$ by the formula

$$
\alpha=\frac{1}{0.5+0.25\left(\mu+\mu^{-1}\right)} \text {. }
$$


The acoustic resonant properties of the FE model were investigated by a broadband frequency pulse. The airflow rate excited the model at the level of the glottis and the acoustic pressure responses were computed at the position of the lips and nose. The power spectral density of the excitation pulse is presented in Fig. 2. The pulse has a flat spectrum in the frequency range up to about $10 \mathrm{kHz}$.

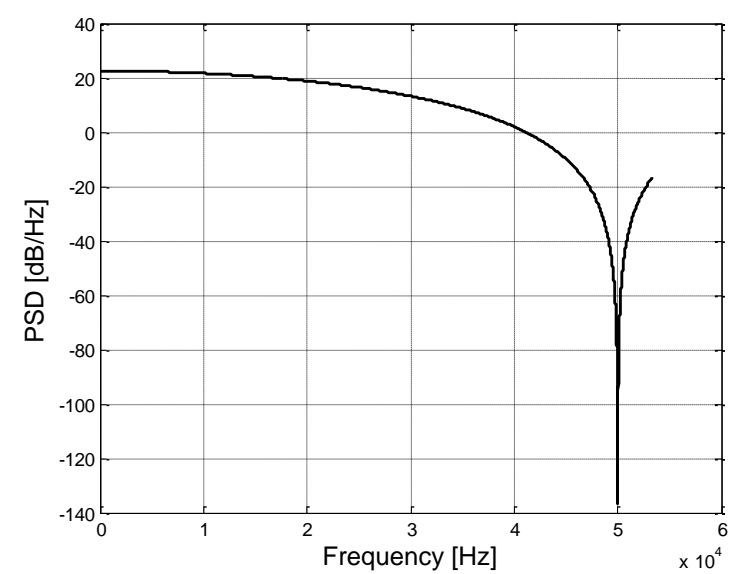

Fig. 2 Exciting airflow pulse in the frequency domain.

\section{Main results}

The character of vibrations of the vocal tract connected with nasal cavities is demonstrated in Fig. 3 .

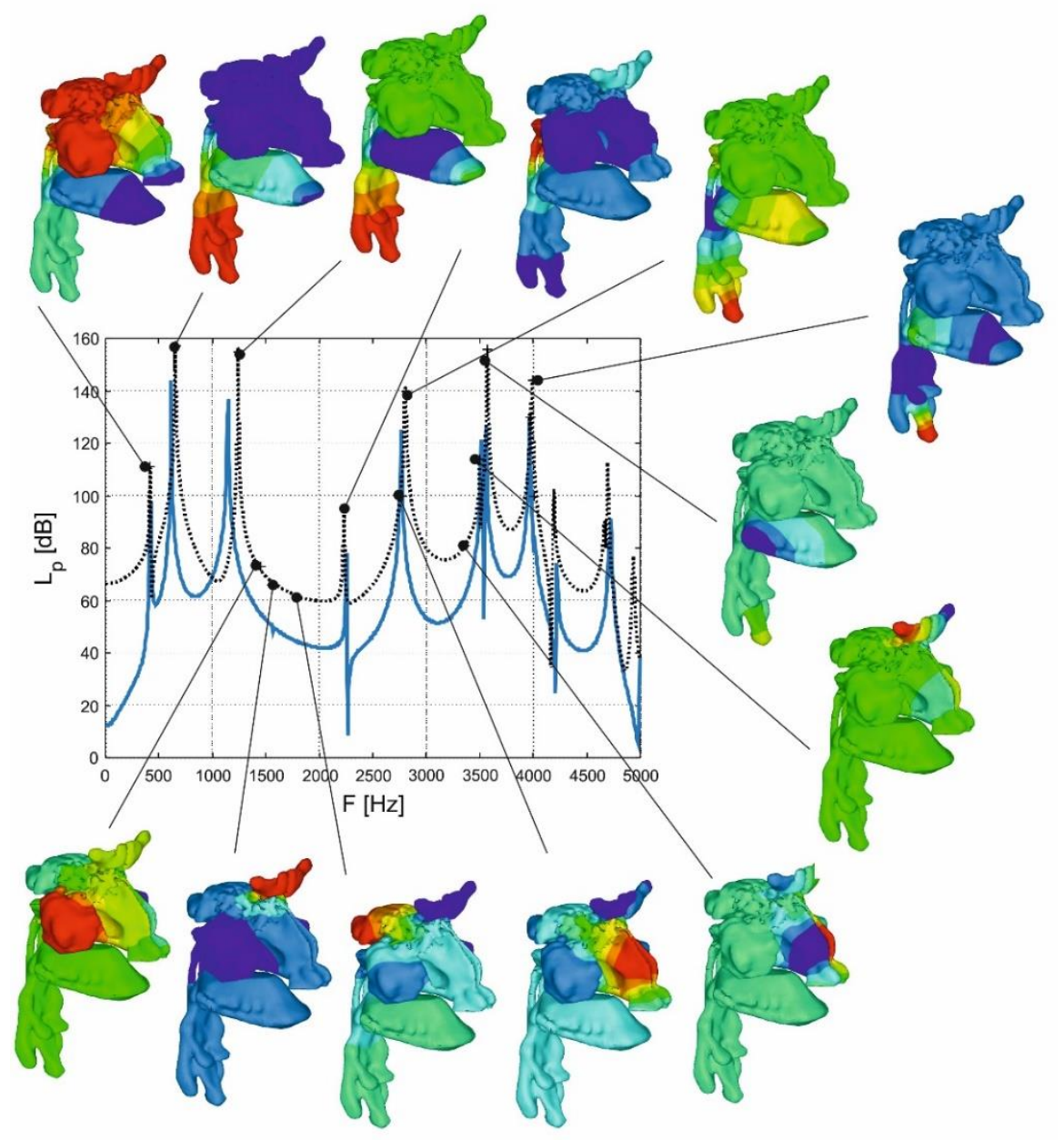

Fig. 3 Acoustic pressure response computed at the lips for the nasalized vowel /a:/ with (full line) and without (dotted line) the lips. 
The effect of the interconnecting the oral and nasal cavities is demonstrated in Fig.4. The new resonances and the antiresonances appear in the output signal. There is one important additional antiresonanceresonance pair at the frequency $\mathrm{F} 1$, naso $\approx 420 \mathrm{~Hz}$ below the first formant frequency $\mathrm{F} 1 \approx 620 \mathrm{~Hz}$ and another antiresonance-resonance pair at $\mathrm{F} 2$, naso $\approx 2250 \mathrm{~Hz}$ between the second $(\mathrm{F} 2 \approx 1150 \mathrm{~Hz})$ and third formant frequency $\mathrm{F} 3 \approx 2780 \mathrm{~Hz}$. The third antiresonance-resonance pair at about $3540 \mathrm{~Hz}$ practically coincides with the formant $\mathrm{F} 4 \approx 3530 \mathrm{~Hz}$. The new antiresonance-resonance pairs can play an important role in the voice quality, because their position can influence the energy in the first four formants.
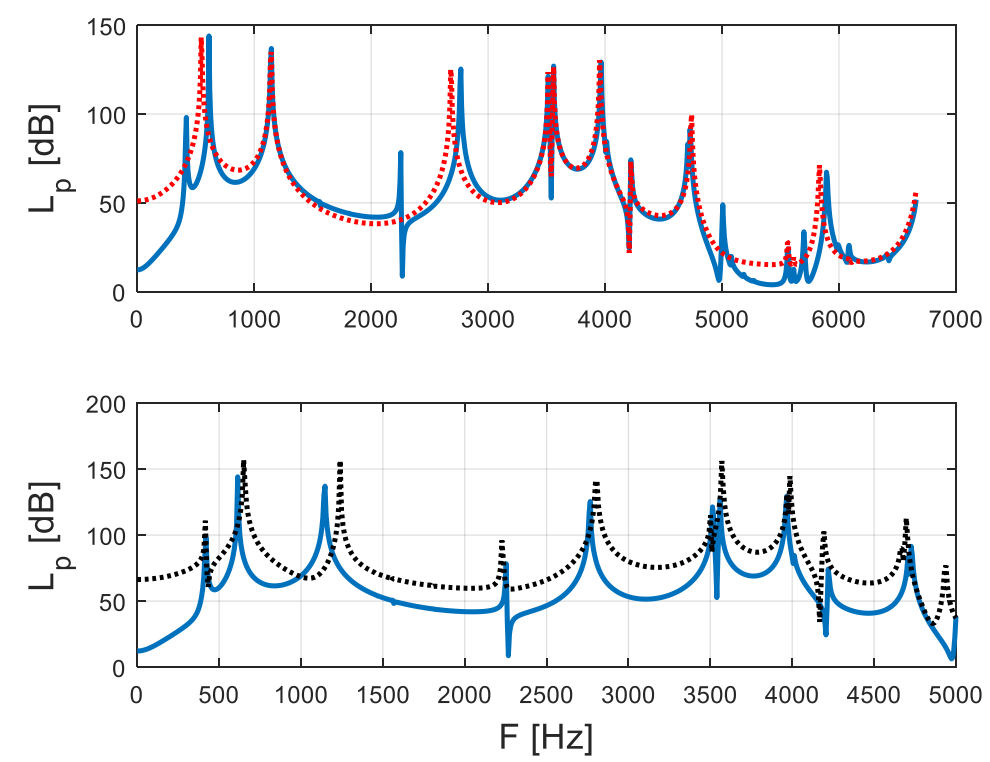

Fig. 4 Power spectral density of the pressure field computed in front of the lips by using the full 3D FE model of the vocal tract without (dotted line) and with the nose cavity (full line), upper panel. Power spectral density of the pressure field computed in front of the lips for model with lips (full line) and without lips (dotted line), bottom panel.

\section{Conclusions}

The results show that the human vocal tract is a very complex resonator. Side branches are generally known to cause antiresonances, i.e., sharp local minima in the resulting transfer function. In speech research the antiresonance phenomenon is well known from the studies of nasalized vowels where the nose acts as the side branch of the vocal tract. The side cavities act as antiresonators which severely decrease the sound level radiating out of the mouth around the antiresonance frequency. Simultaneously, however, they also act as resonators which can amplify the acoustic output at different frequencies. These findings suggest that the side cavities may play a beneficial role in producing the "resonant voice". Formant clustering around 3-4 kHz has been considered to play an important role for the professional speaker's or singer's voice quality.

\section{Acknowledgement}

The study has been supported by a grant from the Czech Science Foundation: No. 16-01246S "Computational and experimental modelling of self-induced vibrations of vocal folds and influence of their impairments on human voice".

\section{References}

Sundberg, J. (1987) The science of the singing voice. DeKalb, Illinois: N. Illinois Univ. Press.

Vampola, T., Horáček, J., Vokřál, J., et al. (2008) FE modeling of human vocal tract acoustics.Part II: Influence of velopharyngeal insufficiency on phonation of vowels. Acta Acustica United With Acustica, vol. 94, pp. 448 460.

Vampola, T., Horáček, J.,. Švec, J.G. (2015) Modeling the influence of piriform sinuses and valleculae on the vocal tract resonances and antiresonances, Acta Acustica United With Acustica, vol. 101, pp. 594-602. 